\title{
Commentary
}

\section{Centralized Electronic Health Records System: A Step Towards an Optimized Health Care Delivery System Ahead of Possible Future Outbreaks}

\author{
Paul Oluwabusayo Omotayo", Joseph Oladimeji Olasupo, Tolulope Benjamin Alegbeleye \\ Department of Pharmacy, Faculty of Pharmacy, University of Ibadan, Ibadan, Nigeria \\ Email address: \\ contact@paulomotayo.tech(P. O. Omotayo),jolasupo977@stu.ui.edu.ng (J. O. Olasupo), balegbeleye937@stu.ui.edu.ng (T. B. Alegbeleye) \\ ${ }^{*}$ Corresponding author
}

\section{To cite this article:}

Paul Oluwabusayo Omotayo, Joseph Oladimeji Olasupo, Tolulope Benjamin Alegbeleye. Centralized Electronic Health Records System: A Step Towards an Optimized Health Care Delivery System Ahead of Possible Future Outbreaks. International Journal of Intelligent Information Systems. Vol. 10, No. 1, 2021, pp. 1-4. doi: 10.11648/j.jiiis.20211001.11

Received: December 14, 2020; Accepted: January 4, 2021; Published: March 10, 2021

\begin{abstract}
COVID-19, the very recent pandemic that brought the global world to its knees has costed the lives of millions, the economy of many nations crippled, a lot of lives distorted due to this unforeseen attack on the health security of the whole world. Outbreaks such as the COVID-19 thrive with increasing mobility all around the world as this novel disease found its way into almost all the nations of the world in less than 6 months. This increases the likelihood for another outbreak of similar nature to occur in the future except certain long-term measures are put in place to strengthen and optimize the health care delivery system and health security. Centralized Electronic Health record system is a core system emanating across developed countries to help improve health system and better health delivery. Apparently, its introduction is somewhat in very few parts of the world, howbeit its full potential is yet to be maximized due to the reluctance of many nations to consider it. In addition, this article highlights the cardinal role of Centralized Health System in surmounting ineffective health care system, highlighting challenges faced by countries faced with the least Human Development Index and discusses why the employment of this system is significant towards achieving an optimized health care system that will be equipped enough to numb the spread of any possible outbreak even before it begins.
\end{abstract}

Keywords: Centralized Electronic Health Records, Health Records, Health Care System, Pandemic, COVID-19

\section{Introduction}

The Health Information Management System Society (HIMSS) defines Electronic Health Record as a lengthwise electronic data of patients' health information obtained during encounters in any health care delivery settings for the purpose of improving quality care. The Information might also include patient demographics, progress notes, problems, lab data, radiology data, etc. [1].

A Centralized Electronic Health Record involves the systemized collection of Patients electronic health information into a centralized database.

The outbreak of COVID-19 triggered the World Health Organization (WHO) as well as affected nations all over the world to roll out certain measures like international travel suspension, supply of personal protective equipment, global public health leadership and multifactorial coordination [2]. These measures have proven to be effective to some extent in curbing the outbreak. Despite these efforts, there seems to be several gaps in achieving optimal global health systems and security.

The increasing influence of digitalization in health system has changed the framework of health care delivery across the countries. Electronic health care records have subsequently replaced the classical paper mechanism of keeping and accessing medical records. Centralized Electronic Health Records is the next step towards achieving cost reduction, reduced medication errors and all-round optimization of the health care system [3]. One of the noteworthy relevance of 
centralized electronic health records is the early symptom detection as a preventive strategy for novel diseases and medical conditions [4]. A revelation of this relevance was established in a report from Wuhan, China. It took 5 days for almost $89 \%$ of patients to be identified and hospitalized as regards to the disease [5]. Early detection in less than 5 days would have had a significant impact in combating the spread of the virus at the early stages. Unfortunately, the Electronic Health Record system was not enhanced enough to address such issue. Implementing a centralized electronic health record system will boost the overall value of EHRs in screening for symptoms and identifying symptomatic patients earlier, thus preventing the fatality of future outbreaks [6].

Centralized EHR validates itself as a crucial system in capturing and identifying people who are more likely to be infected during an outbreak, such as individuals who live in areas with concentrated levels of infection. Migration of individuals to and from these identified places can be checked and controlled to limit widespread transmission of the disease, via the collaborative efforts between the Centralized Electronic Health Records and Health Officials, Immigration Services and Custom Services. Thus, providing sure possibility for a remote communication between the health care sector and other government agencies across states and countries with the aim of limiting widespread of a disease during a pandemic.

As it is a known fact that any bulk of data is irrelevant without proper analysis of the data, definitely, a Centralized EHRs will provide sufficient data with an added advantagethe possibility and ease of identification of uncommon trends and patterns which will help shed light on the rationales for the success and failures of different management therapies when it comes to addressing the symptoms and disease as a whole. Thus, subtle information (such as the effect of genetic variations in response to drugs, efficacy of various herbal medicines, environmental factors effect on drug response, etc.) will be identified, leading to quick detection of evidence-based management of conditions in a pandemic and its implementation to reduce mortality rate and improve quality of lives.

\section{Centralized Electronic Health Record System and Covid-19 Pandemic}

The efficiency of infection prevention lies in the speedy and accurate transfer of medical records between all healthcare personnel associated with a patient's medical state [7]. One of the major responses taken by most countries both developed and developing nations is the tracing, tracking, testing and isolation of susceptible individuals. It was explained how the Albertan Electronic Health Record accessed through the Alberta Netcare portal enables SARS-COV-2 tests results to be available to all authorized custodians and authorized affiliates (i.e. authorized ANP users) in Alberta [8]. The self- assessment online tool developed by the Alberta Health services links each patient's SARS-COV-2 tests results to the Albertan Netcare portal via their Albertan health care number. Therefore, if an Albertan, tests positive for SARS-COV-2, a contact tracer from Albertan health services gets in touch with the infected person using the contact information provided at the testing center to determine others who may have been exposed to the infected person over the previous 21 days. This in turn helps the authorities to carry out efficient contact tracing since the information is readily available to them via the Alberta Netcare portal.

As potent as the different actions taken to flatten the curve are, it cannot realistically account for everyone whose profiles match the requirements of the testing [9]. This gap highlights how countries operating a Centralized Electronic Health Record System identified and spread the surveillance of susceptible citizens through the efficient analytics captured within the database that utilizes all the information of the patient such as reported symptoms, risk scores and underlying conditions. These information aids the accurate prediction of patients with more likeliness to be infected. This fluid passing of information from the community pharmacy, primary health clinics down to the hospital ensures this possibility of better tracking and monitoring. This system provides an integrated, unified, and coordinated network of health services and the availability of such services has been crucial in management of the spread of the pandemic. Information technology, specialized use of data model and the emerging Artificial intelligence are crucial for easy tracking and identification [10].

\section{Factors Limiting Centralized Electronic Health Record}

Howbeit, the effectiveness of this new system is threatened substantially by different factors which includes communication and interoperability, standardization and funding [11]. With particular highlight on the problem of funding in the health care sector all around, World Health Organization recognizes underfunding as the principal block in the attainment of better health care system. The budget pattern distributed in Nigeria health care system from 2017 to 2020 has encountered a downslope coupled with the trouble in conveying basic health delivery. This is seemingly a comparative pattern in most African countries, central and east Africa and in other Asian nations. In as much as initiating, incorporating and developing a centralized electronic health record system guarantees accessible and improved health system delivery later on, it is very unrealistic for countries with lower United Nation Human Development Index to create and define procedures for centralized electronic health records. Likewise, innovation progression over the world which constantly incorporates health administration and records deliver unsafe worry by patients on digital protection of their medical records. The digital danger presented by the expanding dilemma of digitalized actions most importantly 
from cyberthreats, tech firms and others create the requirements for more tight guidelines to oversee, secure and offer patients right and incognito.

\section{The Need for Urgent Implementation of a Centralized Electronic Health Records System}

\subsection{Early Detection and Control of Disease Outbreak}

Infection episodes can be so crippling and this may result to a more prominent mortality and morbidity rate for nations over the globe. Subsequently, models for medical services ought to generally target improving the nature of health outcomes by early identification and detection of novel diseases. Many health conditions have very much been monitored and controlled with a decreased pace of the disease with the aid of the centralized electronic health record system and different algorithms. This is in like manner significant particularly for novel diseases that are obscure, troublesome and difficult to clinicians to diagnose. Thus, efficient utilization of the data obtained from centralized electronic health records systems, Artificial Intelligence and design will help decrease pace of flare-up and better monitoring [12].

\subsection{Improvement in Patient Diagnostics and Health Outcomes}

The success of any therapeutic intervention in the health care setting is very dependent on the efficiency and accuracy of the diagnosis [13]. In avoidance of empirical therapies and blind recommendations, an optimal system must be in place to ensure an excellent outcome from diagnosis. A patient visiting a clinic, hospital or pharmacy to receive any level of health care is at the mercy of accurate diagnosis. However, it takes more than a test to confirm the condition of different patients. This emphasizes why a centralized electronic health records system should be in place, so that previous results and patterns of diagnosis can be made available to any health care provider at any point in time to ensure a successful health outcome [14] Also, the outcome of health care provision is directly affected by the level of accurate decision making, reduced medication error, reduced adverse effects of drugs, etc. all which have been considered and documented to be significantly improved by a centralized electronic health record system.

\subsection{Centralized Electronic Health Records Offer More Convenience to Patients}

Patients are at the core of every health care dispensation; thus, patient satisfaction is very crucial to any claim of therapeutic success. Many patients undergo tremendous amount of stress in the process of seeking for health information; moving from one region to another, making several phone calls, trying hard to remember their medication history. The orthodox Paper based health record system has no doubt mitigated this problem to some extent, but the efficiency of the system and the distance barrier creates a bigger problem for a patient to get specific information from their health records. Information can be generated in less than two minutes within a centralized electronic health records system regardless of the location of the patient [15]. This worrisome series of repetitive tests and procedure, inability to manage geriatric patient medication history attached to old fashioned system will be eliminated leading to a monumental cost saving and an improved convenience in the reception of health care.

\subsection{Centralized Electronic Health Records System Is Capable of Driving the Expansion of Telemedicine}

Telemedicine allows healthcare providers to evaluate, diagnose and treat patients without the need for an in-person visit. Patients can communicate with physicians from their home using available devices and technology. With the adoption of a centralized electronic health records system, patients now have access to medical services from providers and specialist anywhere around the globe. This system enables authorized medical personnel to make informed, science-based decisions that will facilitate faster diagnosing, fewer prescribing errors, and better patient outcomes with comprehensive patient histories and digital images [16].

\subsection{Centralized Electronic Health Records System Will Help Increase Health Care Delivery Systems and Services in Rural Areas}

It is noteworthy to admit to the difficulties in initiating digitalized health system in rural areas but the immense structure of a centralized electronic health records system can help identify major setbacks and track an outbreak of a disease by collating analyzed data from frequently observed symptoms making it easier for governmental agencies and organization to proffer quick aid to such areas.

\section{Conclusion}

This new novel disease, COVID-19, has put countries all around the globe in a terrible dilemma with countries plunged into economic chaos, loss of loved ones and compulsory lockdowns. Although there are reactive measures put in place by several countries to help curb the spread of the virus, however, these measures are insufficient in minimizing the danger and impact of the virus. Centralized electronic health records system, a system that would capture real time data that follow patients across health services and contain all relevant patient-centric and aggregate data, and are shared across services, thus allowing for swift communication among providers [17], will not only improve health care delivery, but will adequately strengthen global public health security. The relevance of a centralized electronic health records system has been well demonstrated over the years. The COVID-19 pandemic has provided significant lessons that are essential towards the optimization of the health care delivery system, one of which includes the importance of centralized electronic health record system, just as proven by Alberta health services. 
A unified adoption of this system will no doubt yield an improved health care system that will shut down any possible outbreak even before it begins.

\section{References}

[1] Mobile Tech Contributions to Healthcare and Patient Experience". Top Mobile Trends. 22 May 2014. Archived from the original on 30 May 2014. Accessed on 24 September, 2020.

[2] Chattu, V. K., Adisesh, A. \& Yaya, S. Canada's role in strengthening global health security during the COVID-19 pandemic. glob health res policy 5, 16 (2020). https://doi.org/10.1186/s41256-020-00146-3

[3] World Health Organization, 2020. COVID-19 reveals gaps in health systems accessed at https://www.weforum.org/agenda/2020/05/covid-19-reveals-g aps-in-public-health-system-who-briefing/ accessed on 30 August, 2020.

[4] Bian, J., Barnes, L. E., Chen, G., \& Xiong, H. (2017, February). Early detection of diseases using electronic health records data and covariance-regularized linear discriminant analysis. In 2017 IEEE EMBS International Conference on Biomedical \& Health Informatics (BHI) (pp. 457-460). IEEE.

[5] Reeves, J. J., Hollandsworth, H. M., Torriani, F. J., Taplitz, R., Abeles, S., Tai-Seale, M., Millen, M., Clay, B. J., \& Longhurst, C. A. (2020). Rapid response to COVID-19: health informatics support for outbreak management in an academic health system. Journal of the American Medical Informatics Association: $\begin{array}{lcr}\text { JAMIA, } & 27 & (6),\end{array}$ https://doi.org/10.1093/jamia/ocaa037

[6] Cohen, A. M., Chamberlin, S., Deloughery, T., Nguyen, M., Bedrick, S., Meninger, S.,... \& Hersh, W. (2020). Detecting Rare Diseases in Electronic Health Records Using Machine Learning and Knowledge Engineering: Case Study of Acute Hepatic Porphyria. medRxiv.

[7] Bali A, Bali D, Iyer N, Iyer M. Management of medical records: facts and figures for surgeons. Journal of maxillofacial and oral surgery. 2011 Sep 1; 10 (3): 199.
[8] Baumgart D. C. (2020). Digital advantage in the COVID-19 response: perspective from Canada's largest integrated digitalized healthcare system. NPJ Digital Medicine, 3 (1), pp. $1-4$.

[9] Center for Disease Control and prevention. Testing for Covid-19 (Online). https://www.cdc.gov/coronavirus/2019-ncov/symptoms-testin g/testing.html. Accessed on the 7th December, 2020.

[10] Ganasegeran K, Abdulrahman SA. Artificial intelligence applications in tracking health behaviors during disease epidemics. InHuman Behaviour Analysis Using Intelligent Systems 2020 (pp. 141-155). Springer, Cham.

[11] Fragidis L. \& Chatzoglou. P (2012). Challenges in implementing nationwide electronic health records: lessons learned and how should be implemented in Greece. 10th international conference on Information Communication Technologies in Health.

[12] University of Scranton Resource Center, 2020. 7 benefits of electronic health records for hospital accessed at https://elearning.scranton.edu/resource/health-human-services/ 7-benefits-electronic-health-records on 22nd November, 2020.

[13] Waller KC, Fox J. Importance of Health History in Diagnosis of an Acute Illness. The Journal for Nurse Practitioners. 2020 Feb 28.

[14] Hillestad, R., Bigelow, J., Bower, A., Girosi, F., Meili, R., Scoville, R., \& Taylor, R. (2005). Can electronic medical record systems transform health care? Potential health benefits, savings, and costs. Health affairs, 24 (5), 1103-1117.

[15] Aljarullah, A., \& El-Masri, S. (2013). A novel system architecture for the national integration of electronic health records: a semi-centralized approach. Journal of medical systems, 37 (4), 9953.

[16] Dorsey, E. R., \& Topol, E. J. (2020). Telemedicine 2020 and the next decade. The Lancet, 395 (10227), 859.

[17] Hoechsmann, A. (2012). Centralized electronic health records benefit emergency medicine. CMAJ, 184 (1), 74-74. 